\title{
ORT.28 - Biological relevance of A2a receptor in Schwann cells infected by Mycobacterium leprae: a possible pathway involved in leprosy?
}

\author{
Plínio Marcos Freire dos Santos ${ }^{1 *}$; Bruno Mietto ${ }^{1}$; Luisa Gutierres ${ }^{1}$; Antônio Marcos Rodrigues \\ Pereira $^{1}$; Chyntia Carolina Diaz Acosta ${ }^{2}$; Maria Cristina Vidal Pessolani ${ }^{1}$; Márcia de Berredo \\ Pinho Moreira ${ }^{1}$. \\ 1 Fiocruz - Fundação Oswaldo Cruz; \\ 2Instituto de Investigaciones en Ciencias de la Salud, UNA, Asunción, Paraguay.
}

Introduction: Leprosy is a chronic infectious disease caused by Mycobacterium leprae which evokes a strong inflammatory response and leads to nerve damage. Two important aspects that favor the establishment of infection and survival of $M$. leprae are: lipid droplet accumulation and the capacity to induce rapid demyelination after extracellular binding to myelinating Schwann cells (SC). Recently, purinergic receptors have been shown to participate in myelination processes, lipid metabolism and immune response. In addition, the purinergic signaling system plays an important role by modulating inflammatory and immune responses, via extracellular adenine nucleotides and their derived nucleoside adenosine (ADO). The enzymatic activities of CD39, CD73 and Adenosine deaminase play strategic roles in regulating purinergic signals through the conversion of ADP/ATP to AMP, AMP to adenosine and adenosine to inosine respectively.

Objective: This study aims to evaluate the role of the purinergic signaling pathway in the pathogenesis of M. leprae in Schwann cells, analyzing the influence of infection on the different components of this pathway, in particular the A2a receptor.

Methodology: To get the results we used Schwann cells and infection of Mycobacterium leprae. Ecto-NTPDasic and Ecto-5'Nucleotidasic activity assays were conducted and to corroborate then we performed Immunofluorescence assay in of the CD39, CD73 and ADA and A2a evaluated by immunofluorescence. Immunocytochemistry with the drugs adenosine, CGS21680, ZM 241385, was performed to observe the production of lipid body, in bacterial viability and cytokine production analysis. Finally we performed fluorescence microscopy to analyze destruction os myelin in primary Shwann cells.

Results: Our data have shown that ML infection increases the activity and the expression of the enzymes CD39 and CD73, ectoenzymes that hydrolyzes extracellular AMP to ADO. In expression of $\mathrm{ADO}$ receptors we have observed that $\mathrm{SC}$ expresses only A2ar and A2br, but the infection decreases the expression of A2ar. We observed the production of cytokine IL- 6 and the result showed that the drugs ADO and CGS, specific agonist of A2a receptor, , increases the secretion of IL- 6 besides decreasing the viability of the bacteria on infection. Finally, using primary cells, the specific agonist of A2a was able to protect myelin, on the other hand the antagonist of receptor was able to destroy the myelin.

Conclusion: the results reveal the involvement of the purinergic system in the infection of SC by $\mathrm{ML}$ reinforcing the role of this pathway in the leprosy pathogenesis, opening a new way for a possible participation of this receptor in ML-induced demyelination.

Keywords: Purinergic Signalling; Shwann cells; Mycobacterium leprae 